\title{
Untersuchungen iiber den Einfluss des Sympathicus auf den Muskeltonus an den tonischen Augenreflexen des Kaninchens.
}

\author{
Von Dr. S. Fujimori. \\ (Aus dem Physiologischen Institut der Universität Bern.)
}

Seitdem durch die Arbeiten von Boeke (Internat. Monatsschr. f. Anat. u. Physiol. 28, 377, 1911; Anat. Anz. 35, 193, 1910; 44, 343, 1913) und Agduhr (Kon. Akad. von Wetensch. Amsterdam, 2 Sectie, Deel 2, Nr. 6, 1920; Kon. Akad. v. Wetensch, Amsterdam, 27, 930, 1919. Ref. Zeitschr. f. d. ges. Neurol. u. Psychiatrie 20, 471, Referate), die den anatomischen Nachweis einer zu der gewöhnlichen motorischen Innervation der willkürlichen Muskeln hinzutretenden besondern Innervation erbracht haben und seitdem Jakob von Uexküll (Umwelt und Innenwelt der Tiere, Springer, Berlin, 1904; Zeitschr. f. Biol. 39, 1900; 58, 305, 1912) das tatsächliche Vorkommen einer eigenartigen Sperrmuskulatur entdeckt hat, und nachdem schliesslich durch $\mathrm{Pe}-$ kelharing (Zeitschr. f. physiol. Chem. 75, 207, 1911; Zeitschr. f. physiol. Chem. 64, 262, 1910; 69, 305, 1910) und Rieser, O. (Arch. f. exp. Pathol. u. Pharmakol. 80, 183, 1917; Pflügers Arch. d. ges. Physiol. 190, 137, 1921 ; Klin. Wochenschr. 1922, S. 1317, 1374) Stoffwechselvorgänge im Muskel näher untersucht worden waren, die nicht mit der gewöhnlichen Tätigkeit des Muskels in einen engeren Zusammenhang zu bringen waren, ist die Untersuchung eines etwaigen Einflusses sympathischer und parasympathischer Nerven auf den Muskel Gegenstand zahlreicher Untersuchungen geworden. Eine gute Uebersicht über die umfangreiche Literatur bis zum Jahre 1923 gibt das Werk von Spiegel (Physiologie und Pathologie des Skelettmuskeltonus, Berlin, Springer, 1923). Trotz der zahlreichen Arbeiten ist die Sachlage noch wenig geklärt, vor allem deshalb, weil die Befunde der einzelnen Autoren nicht miteinander übereinstimmen.

Scheinbar am klarsten liegen die Ergebnisse bei den jenigen Untersuchungen, wo pharmakologische Mittel herangezogen wurden, um eine Entscheidung über etwaige autonome Innervation herbeizuführen. Es scheint kein Zweifel zu sein, dass die sogenannten parasympathisch erregenden Gifte einen erregenden Einfluss auf die Will- 
kürmuskulatur besitzen, in besonders ausgesprochener Weise nach Beseitigung der Cerebrospinal-Innervation, und dass anderseits die parasympathisch lāhmenden Gifte antagonistisch wirken. Auch einige der sympathischen Gifte äussern eine Wirkung auf die willkürliche Muskulatur. Aber gerade diese pharmakologischen Versuche können wenig zur entscheidenden Klärung beitragen, weil die theoretische Basis derselben eine recht unsichere ist. Magnus hat wiederholt darauf hingewiesen, dass durch Giftversuche eine unbekannte Innervation bezw. ein unbekannter Innervationsmechanismus nicht nachgewiesen werden kann, weil man mit zwei Unbekannten arbeitet: die unbekannte Innervation und die unbekannte Wirkungsweise des angewandten Giftes. Neuere Arbeiten des Berner Physiologischen Instituts, diejenigen von Spycher (Zeitschr. f. Biologie, 1922), von Benteli und Scheinfinkel (letztere beiden noch nicht veröffentlicht) haben durch Anwendung einer neuen Methode beweisen können, dass die primāre Wirkung der sogenannten autonomen Gifte eine Beeinflussung physikalischchemischer Zeitreaktionen im Muskel ist. Mit diesem Nachweis fällt aber die Möglichkeit, einen gesicherten Nachweis autonomer Innervation mit Hilfe pharmakologischer Stoffe zu liefern, dahin, selbstverständlich wie vor ein Parallelismus zwischen pharmakologischer Giftwirkung und autonomer Innervation bestehen, aber derselbe ist kein zwangsläufiger, sondern ein solcher von sekundärer Natur. Bei dieser Sachlage ist man auf andere experimentelle Methoden angewiesen. Diese haben aber wie gesagt, $z u$ widersprechenden Resultaten geführt. Eine grosse Anzahl von Arbeiten haben ergeben, dass, ob ein Gebiet im Besitze seiner sympathischen Innervation ist oder nicht, keinen erkennbaren Unterschied mit sich bringt. Diese Autoren führen daher die positiven Beobachtungen der andern Autoren auf Versuchsfehler oder auf andere sekundäre Momente zurück. Die Versuchsfehler können darin bestehen, dass bei den Operationen die zur Entfernung der sympathischen Innervation dienen, benachbarte motorische Nerven beschādigt werden, sodass temporāre Symptome in der von ihnen innervierten Muskulatur auftreten. Sodann kann die durch Entfernung der sympathischen Innervation geānderte Gefāssinnervation Kreislaufsverānderungen mit sich bringen, welche gleichfalls Symptome der Tonusveränderungen vortāuschen können. Ich beschrānke mich bei meiner Diskussion auf die Erörterung der Verhältnisse des Sympathicus, weil meine eigenen Untersuchungen sich ausschliesslich auf die sympathische Innervation beziehen und die anatomischen Nachweise bisher ausschliesslich auf solche von sympathischer Natur sich beziehen. Aus dem Berner Physiologischen Institut sind gleichfalls eine Reihe von Untersuchungen hervorgegangen, die sich mit dem Probleme der autonomen Innervation des Muskels befassen. Auch die Ergebnisse dieser Arbeiten 
sind nicht eindeutig ausgefallen. Maumary exstirpierte beim Frosch die sympathischen Nerven, welche die eine Extremität versorgen und fand mit Hilfe von Apparaten, die er zu Zwecken der Spannungsmessung konstruiert hatte, unterschiede in der sympathicuslosen und normalen Muskulatur. Auch beobachtete er Erscheinungen, welche darauf deuteten, dass die Folgen der halbseitigen Entfernung des Labyrinths und der Entfernung der sympathischen Innervation additiv sich zusammensetzen. Schmid, der am Kaninchen arbeitete, konnte nach Exstirpation sämtlicher sympathischer Ganglien, von denen die sympathischen Nerven zu einer hintern Extremität ausgehen, keinen Unterschied in den mechanischen Verhältnissen des Muskels und in der von ihm eingehend untersuchten Nichtermüdbarkeit der Muskulatur feststellen. Nur ganz im Anfang fand sich ein gewisser Tonusverlust, der aber sehr bald zurückging. Mosca [Berner Doktordisseratation; die Befunde desselben sind in der Arbeit von Schmid (Zeitschr. f. Biologie, 1922) mitgeteilt] untersuchte die Haltung des Kaninchenohres und verglich die Seite mit und ohne sympathische Innervation. Dieses Objekt war deshalb günstig, weil sich die Entfernung des Sympathicus ohne jede Schädigung der motorischen Intervation der betreffenden Muskulatur ausführen liess. Es ergab sich, dass auf derjenigen Seite, wo der Sympathicus fehlte, ein Gewicht, welches auf der normalen Seite das in die Höhe stehende Ohr in seiner Lage nicht änderte, das Ohr herabzog. Es bestand somit ein deutlich erkennbarer Tonusverlust, der viele Wochen lang, wenn auch mit abnehmender Stärke, beobachtet werden konnte. Aber diese Beobachtung eines Tonusunterschiedes gelang nur unter einer wesentlichen Bedingung, nämlich, dass die Aufmerksamkeit des Tieres in keinerlei Weise geweckt wurde. Sobald nämlich durch irgend etwas die Aufmerksamkeit des Tieres beansprucht wurde, schwand jeder Tonusunterschied. Diese Beobachtung scheint Anhaltspunkte zu enthalten, welche geeignet sind, die grosse Schwierigkeit zu erklären, mit welcher die Feststellung eines von Sympathicus abhängigen Tonus der Muskulatur behaftet sein muss, selbst wenn sie wirklich vorhanden ist. Es ist klar, dass was Tonus genannt wird, von der gewöhlichen motorischen Innervation gleichfalls geleistet wird und wir mit mechanischen Methoden keinerlei Mittel besitzen, um den Tonusanteil, der etwa vom Sympathicus herrühren kōnnte, von demjenigen zu unterscheiden, der vom cerebrospinalen Systeme herrührt. Die einzige Möglichkeit bestände darin, dass die Willensimpulse möglichst ausgeschaltet werden und nicht allein die Willensimpulse, sondern auch diejenigen, welche fortwährend von niederen Hirnteilen und vom Rückenmark auf die Muskulatur übergehen. Die Extremitäten sind von vornherein ein denkbar ungünstiges Objekt, da dieselben fortwährend für die normale Haltung des Tieres beansprucht wer- 
den und jederzeit ihnen Impulse vom Rückenmark zufliessen. Das Ohr ist hierin günstiger gestellt und deshalb sind die Untersuchungen von Mosca bisher die einzigen, welche mit einiger Wahrscheinlichkeit zu Gunsten einer sympathischen Beeinflussung der Spannungsverhältnisse des Muskels sprechen. Abcr es ist nur eine Wahrscheinlichkeit. Es ist daher geboten, weiteres Material zu sammeln. Ein günstiges Objekt, um nach den soeben angedeuteten Gesichtspunkten einen etwaigen Einfluss des Sympathicus auf die quergestreifte Muskulatur zu untersuchen, liegt in den Augenmuskeln des Kaninchens vor, und ich folgte daher gern der Anregung von Prof. Asher, Versuche darüber anzustellen, ob Augennuskeln mit und ohne sympathische Innervation irgend einen Unterschied in ihren Leistungen aufwiesen.

Die Gründe, weshalb die Augenmuskeln des Kaninchens günstige Objekte für das in Frage stehende Problem sind, sind die nachfolgenden: 1. Die Augenmuskeln des Kaninchens sind in einem weitgehenden Umfang voin Willen unabhängig; das Kaninchen führt keine Blickbewegungen aus, wie wir das vom Menschen kennen. 2. Die sympathische Innervation dieser Muskeln liegt in ihren Ursprüngen weit getrennt von ihrer motorischen Innervation, sodass bei der Operation eine Beschädigung der letzteren gar nicht in Frage kommt. 3. Sind gerade die Augenmuskeln der Ort ausgeprägter tonischer Reflexe. 4. Wir besitzen für die Untersuchungen tonischer Reflexe vorzügliche Methoden, die von Magnus und seinen Mitarbeitern ausgebildet worden sind. Die Augenmuskeln haben bisher nur in einer Arbeit von Köllner und Hoffmann (Archiv f. Augenheilkunde, Bd. 90, 1922, S. 170) zur Prüfung der Frage, ob die quergestreifte Muskulatur ausser der gewöhnlichen tetanischen Tätigkeitsform noch eine andere, der glatten Muskulatur entsprechende, zukāme, gedient. 'Die Autoren bedienten sich hierzu der Registrierung der Aktionsströme der Augenmuskeln. Es ergab sich kein Anhaltspunkt dafür, dass die vestibüläre Innervation, von welcher die tonischen Reflexe ausgehen, irgendwie erkennbar, anders in den Aktionsströmen zur Geltung käme. Diese Untersuchung unterscheidet sich insofern von der meinigen, als sie nicht Bezug ninımt auf die eigentliche sympathische Innervation.

Meine Aufgabe bestand darin, gut untersuchte und möglichst analysierte tonische Reflexe der Augen zu prüfen, einmal bei Tieren, welche im Besitz der normalen sympathischen Innervation waren, anderseits bei Tieren, denen diese Innervation fehlte. Besonders günstig ist bei dieser Untersuchung der Umstand, dass dieselbe sich an einem und demselben Tiere zu gleicher Zeit anstellen lässt, indem nur einseitig der Sympathicus exstirpiert wird, während das andere Auge als Kontrollauge dient.

Ich beginne die Besprechung der Methodik mit der Angabe iber die Art und Weise, wie die Exstirpation des 
Sympathicus geschah. Alle Operationen wurden in Morphiumnarkose ausgefuhrt. Der Sympathicus wurde auf der linken Seite exstirpiert. Um sicher zu sein, dass jede kopfwårtsziehende Innervation ausgeschlossen wurde, habe ich nach Freilegung des Halssympathicus denselben sowohl nach unten wie nach oben verfolgt, bis ich zum Ganglion cervicale inferius und Ganglion cervicale superius vorgedrungen war. Sodann hahe ich beide Ganglien vollståndig exstirpiert. Der Erfolg der Exstirpation war sofort an dem Ohre und der Pupille der gleichen Seite zu beobachten. Die Wunde heilte per prima.

Meine erste Versuchsreihe befasste sich mit den tonischen Labyrinthreflexen auf die Augen, welche durch die verschiedene Lage des Kopfes zum Raume ausgelōst werden. Diese tonischen Labyrinthrefiexe sind in mustergültiger Weise durch Magnus und seine Mitarbeiter untersucht und analysiert worden und ich bin den methodischen Anweisungen dieser Autoren genau gefolgt. Was die Untersuchung der Stellung der Lage anbetrifft, so habe ich dieselbe in der Art und Weise durchgeführt, wie das von Hoeve und de Kleijn (PAligers Arch. Bd. 169, 1917, S..241) und von de Kleijn und Magnus (Pflügers Arch. Bd. 168, 1920, S. 179) genau beschrieben worden ist. Um die Lage des Kopfes im Raume zu verăndeın, stand mir ein Operationstisch des Berner Physiologischen Instituts zur Verfügung, der ein Kugelgelenk besass, sodass ich das auf dem Kaninchenbrett aufgebundene Kaninchen sowohl gegen die Horizontale neigen konnte, wie auch in Scitenwendung bringen konnte. Den Winkelgrad, in welchem die Drehung stattfand, habe ich auf die Weise gemessen, dass ich an dem Tisch eine Winkelteilung anbrachte, uber welcher ein loth hing. Die Abweichung des Lothes von der Nullteilung der Winkelteilung ergab den Winkel, um welchen ich gedreht hatte. Genau, wie es im Utrechtex Laboratorium üblich ist, habe ich auf die Hornhăute des Kaninchens mit Bleiacetat ein Kreuz eingebrannt und im Auge einen Rahmen sich spiegeln lassen, in dem ein vertikaler und horizontaler Faden eingespannt war. Dieser Rahmen wurde fest mit dem Tierhalter verbunden und die wechselnde Stellung des auf der Hornhaut eingebrannten Kreuzes zu den fixen Faden als Mass der Raddrehung und der Hőhenabweichung benutzt. Der photographische Apparat wurde gleichfalls auf dem Operationstisch, der zur Drehung diente, befestigt. Die einzige Abweichung von den Anweisungen der Utrechter Forscher war dadurch bedingt, dass ich die Stellung beider Augen gleichzeitig zu bestimmen hatte. Um dies zu ermőglichen, habe ich mich des Asher'schen Winkelspiegels, wie er in der Arbeit von Car lo Schneider (Biochem. Zeitschr. 1922, Bd. 133) beschrieben worden ist, bedient. Beide Augen waren gleichzeitig in den beiden Schenkeln des Winkelspiegels sichtbar. Der Winkelspiegel musste sehr gut beleuchtet werden und dann wurden die Bilder der beiden Augen photographiert. Da das Licht gleichmåssig und sehr günstig sein muss, sind die Aufnahmen manchmal mit gewissen Schwierigkeiten verknüpft. Die gelungenen Photographien zeigen in sehr deutlicher Weise die bestehenden Verhaltnisse.

Zuerst habe ich Untersuchungen des normalen Tieres vorgenommen, um iber dessen tonische Labyrinthreflexe auf die Augen unter den von mir benutzten experimentellen Bedingungen genau informiert zu sein. Da meine Hauptaufgabe in einer Vergleichung bestand, habe ich mich mit der genauen Untersuchung von je 5 Lagen begniigt, nămlich der normalen Bauchlage, und vier Wendungen um je $45 \mathrm{Grad}$. In der zweiten Reihe habe ich dann vorher die sympathische Innervation des linken Augen entfernt, und dann erneut untersucht. Der Gedanke, der mich hierbei leitete, war der, dass falls der Sympathicus auf die tonischen Reflexe des Auges einen Einfluss hătte, das Fehlen dieses Einflusses sich möglicherweise in den vom Labyrinth ausgelösten tonischen Reflexen zeigen kőnnte. Nachdem diese Versuchsreihe abgeschlossen worden war, habe ich das Labyrinth exstirpiert. Hiexdurch andern sich die tonischen Labyrinthreflexe, und wiederum waren Bedingungen geschaffen, um eine etwaige von sympathischer Innervation herrithrende Komponente zum Ausdruck gelangen zu lassen.

Die Resultate dieser drei Versuchsreihen habe ich in den Tabellen A, B, C und 
D niedergelegt. Die Ergebnisse, welche an 5 bewz. 6 Tieren gewonnen worden sind, sind durchaus eindeutig, sodass sie nicht vieler Worte bedürfen. Irgend einen Einfluss des Fehlens der sympathischen Innervation auf die tonischen Labyrinthreflexe, welche durch eine verschiedene Lage des Kopfes im Raume ausgelöst werden, ist nicht erkennbar. Dieses Ergebnis spricht gegen die Annahme, dass die sympathische Innervation einen Einfluss auf tonische Kontraktionen der Augenmuskulatur habe. Gerade bei dieser Art von Kontraktion wäre ja ein Einfluss des Sympathicus zu erwarten gewesen. Das negative Eıgebnis ist um so bemerkenswerter, als es sich um diejenigen Muskeln handelt, bei welchen Boeke das Vorhandensein sympathischer Innervation anatomisch nachgewiesen hat. Es erhebt sich die Frage; ob mein negativeş Ergebnis streng beweisend für das Nichtvorhandensein sympathischer Innervation der Augenmuskeln ist. Ein Einwand, der erhoben werden könnte, ist der, dass die Augenmuskeln ihre sympathischen Nerven gar nicht vom Halssympathicus erhalten. Dieser Einwand

Tabelle A. Untersuchung über tonische Labyrinthreflexe an den Augen.

Prüfungsmethode.

Beide Augen gleichzeitig mit dem Winkelspiegel bei folgender 5 Lagen photographiert.

1. Das Tier ist in Bauchlage, Mundspalte horizontal, und Tierbrett horizontal.

2. Das Tier ist, den Kopf nach rechts unten, $45^{\circ}$ um die Sagittalachse gedreht worden.

3. Das Tier ist, den Kopf nach links unten, $45^{\circ}$ um die Sagittalachse gedreht worden.

4. Das Tier ist, den Kopf nach oben, $45^{\circ}$ um die bitemporale Achse gedreht worden.

5. Das Tier ist, den Kopf nach unten, $45^{\circ}$ gedreht worden.

Die Winkeldifferenz $-x^{\circ}$ ist die Raddrehung der Augen nach dem Schwanz, und $+x^{\circ}$ ist die Drehung nach dem Kopf.

Die Höhendifferenz $+x \mathrm{~mm}$ ist die Zunahme der Distanz des Mittelpunktes der Cornea von der Lăngsund Quer-Linie. $-x$ ist Abnahme. $(x=1 / 2$ m.m. $)$
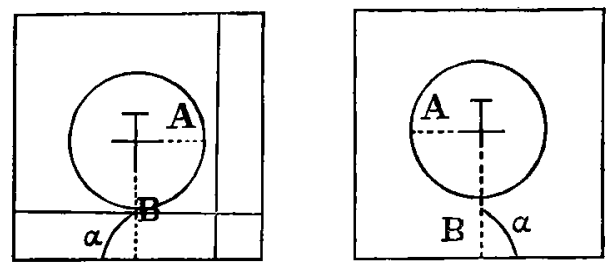
A, B. Distanz des Mittelpunktes der Cornea von den beiden Linien.

๙. Winkel.

Die Photographie geschah in folgender Reihenfolge :

I. normales Tier.

II. links sympathicusloses Tier.

III. rechts labyrinthexstirpiertes Tier (links Sympathicuslos). 1-2 Wochen liegen zwischen II. u. III. Operation. 


\begin{tabular}{|c|c|c|c|c|c|c|}
\hline 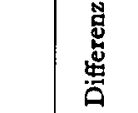 & $\begin{array}{l}\text { मक न न } \\
1+1\end{array}$ & $\begin{array}{l}\infty+H_{N} \\
+1+1\end{array}$ & $\begin{array}{c}\text { m음욮 } \\
1+1\end{array}$ & 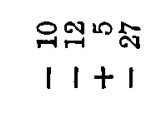 & $\begin{array}{l}\operatorname{man}_{-1} \\
1+1\end{array}$ & $\begin{array}{l}m \infty \infty \frac{5}{\omega} \\
+1+1\end{array}$ \\
\hline 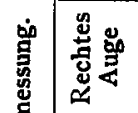 & 융 & 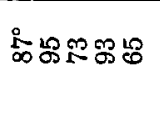 & 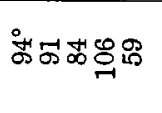 & 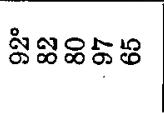 & 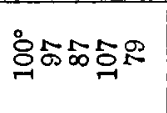 & 号志品昌尔 \\
\hline 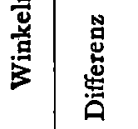 & 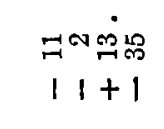 & $\begin{array}{l}\approx+{ }^{H} \\
11+1\end{array}$ & $\begin{array}{l}\text { 두ㅇㅝㅠ } \\
1++1\end{array}$ & $\begin{array}{l}\infty \sim \infty \\
11+1 \\
1+\infty\end{array}$ & $\begin{array}{c}\operatorname{mos} 9 \\
11+1\end{array}$ & $\begin{array}{l}\text { 유ㅇㅛㅛ } \\
1++1\end{array}$ \\
\hline 造 & 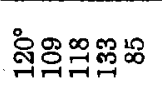 & 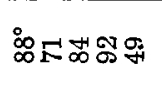 & 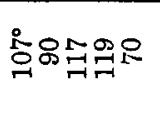 & 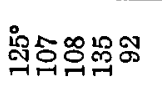 & 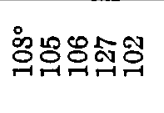 & 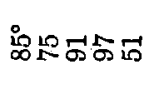 \\
\hline N & . . & $000 \stackrel{0}{\mathrm{i}}$ & 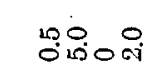 & 0000 & & 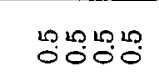 \\
\hline 承 & $11+$ & $t$ & +++ & $1+$ & $1+t$ & $+11+$ \\
\hline 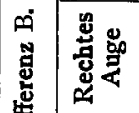 & 잉ำ & 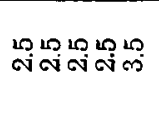 & 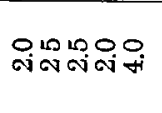 & 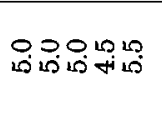 & 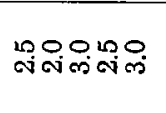 & ن \\
\hline 宽 & om이물 & 10 000 & 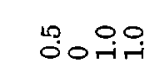 & مُ0 & :000 & $00 \stackrel{100}{\circ}$ \\
\hline 虫 & $11+$ & $1+$ & $11+$ & $+1+$ & +1 & $1+$ \\
\hline 占品 & $\begin{array}{l}0.9000 \\
601010\end{array}$ & 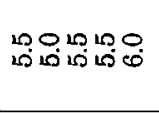 & 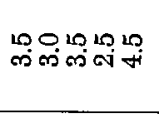 & 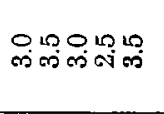 & فم & 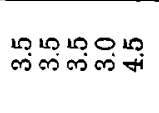 \\
\hline N & No & مُ & تُ & فُ & 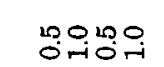 & 19090 \\
\hline 营 & $+1+$ & $+1+$ & $+1+$ & $+11+$ & $+11+$ & +1 \\
\hline 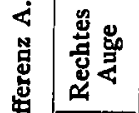 & 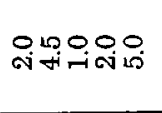 & 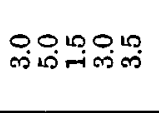 & 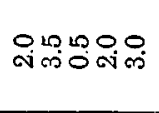 & 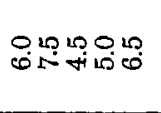 & مَّ & fif \\
\hline 䔅 & ำ & تج & $\begin{array}{l}100100 \\
0 \times 10\end{array}$ & مأ & Orioo & 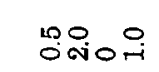 \\
\hline 莒 & $1+t$ & $1+t+$ & $1+t+$ & $1+t$ & $1+$ & $1+t$ \\
\hline 咅 & فِ & 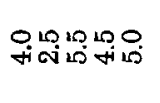 & $\begin{array}{l}0.10010 \% \\
\text { in }\end{array}$ & 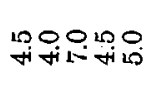 & 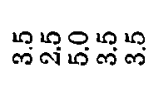 & 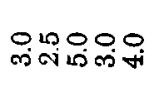 \\
\hline 品 & ーNのみ & TONCOA H & HNO & ת1 & Hળ円 & مג \\
\hline 总兽 & $\mapsto$ & ヨ & 可 & 己 & $>$ & $\stackrel{5}{>}$ \\
\hline
\end{tabular}




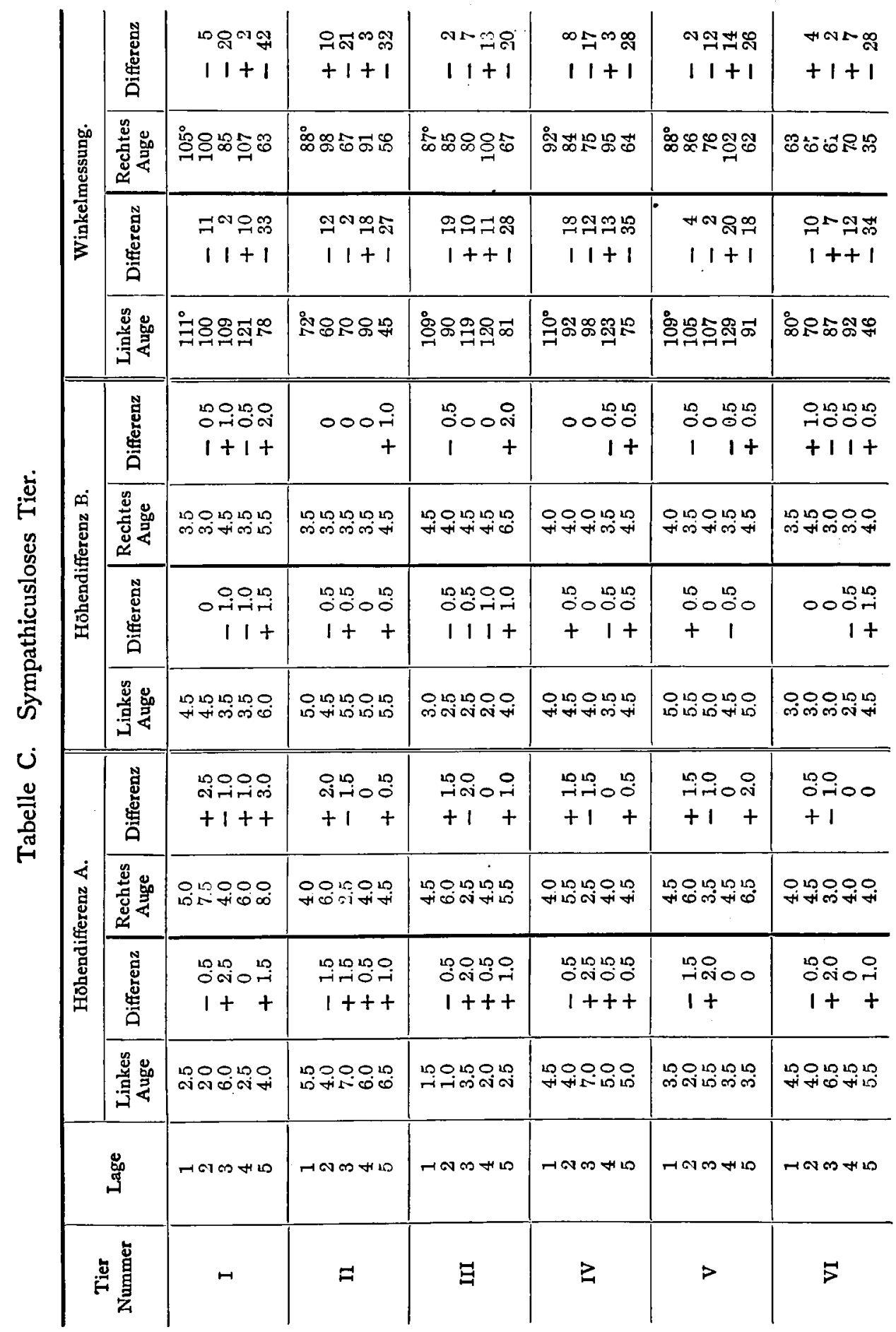




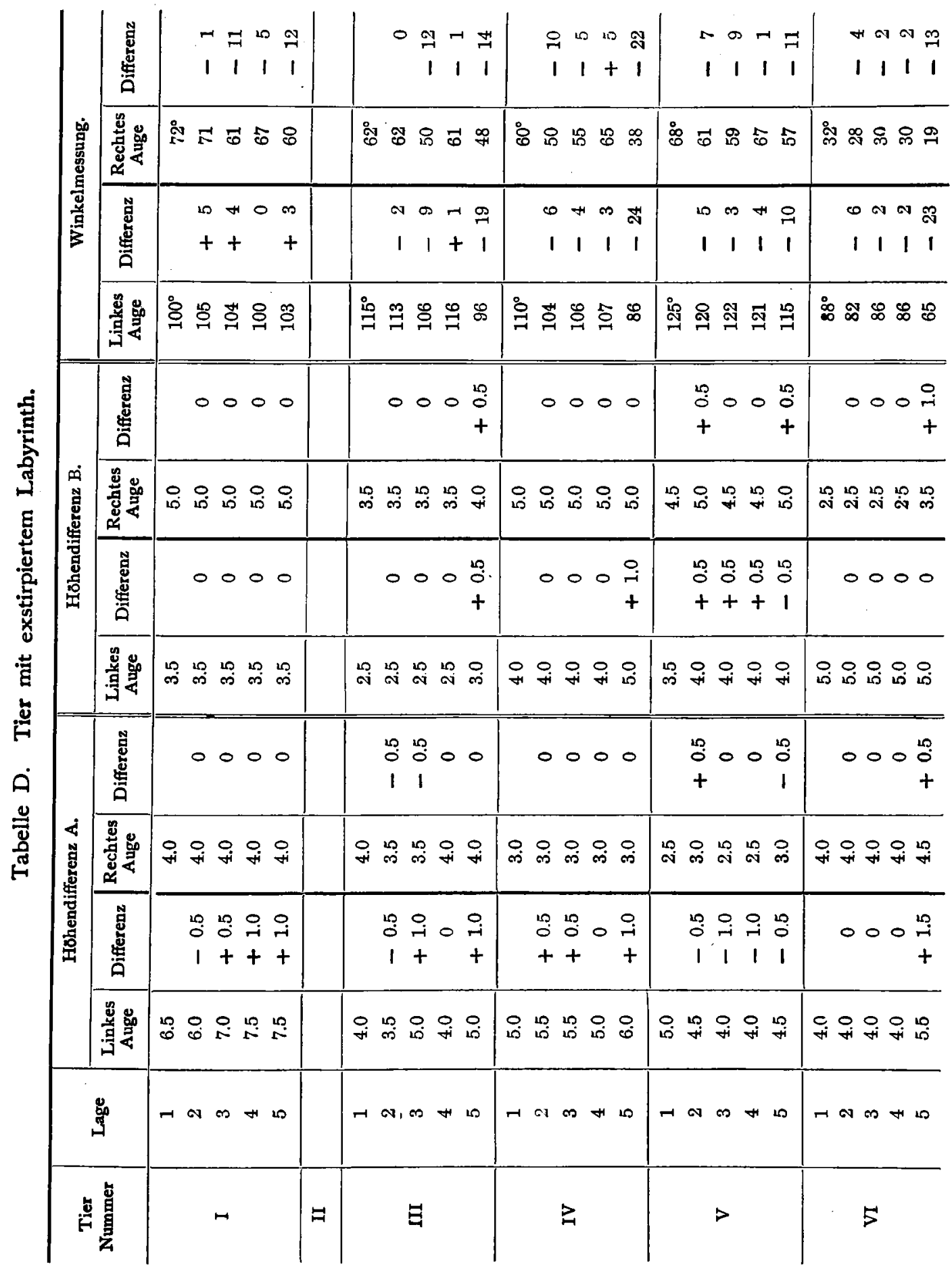


ist natürlich rein hypothetischer Natur: denn alles, was wir anatomisch wissen, belehrt uns darüber, dass die Teile des Kopfes, welche sympathische Innervation erhalten, sie auf dem Wege von Nerven erhalten, die ihren Ursprung von den obern Thorakalsegmenten nehmen und aut dem Wege des Halssympathicus nach dem Kopfe gelangen. Erst wenn anatomiseh ein anderer Weg zu den Augenmuskeln gefunden würde als Fasern, die entweder vom Ganglion cervicale inferius oder Ganglion cervicale superius ausgehen, wäre der Einwand ernstlich ins Auge zu fassen. Es ist ein anderer Einwand möglich, und das ist der, dass die von Boeke gefundenen akzessorischen Nerven nicht sympathischer, sondern nach den Anschauungen von E. Frank parasympathischer Natur wären. Wenn dies der Fall wäre, so kann natürlich die Exstirpation des Halssympathicus und seiner Ganglien keinen Finfluss ausüben. Die etwaige parasympathische Innervation der Augenmuskeln ist ein anderes Problem als das von mir untersuchte. Meine Fragestellung betrifft ausschliesslich die sympathische Innervation. Ein anderer Einwand, der möglich ist, wäre der, dass zwar die tonischen Labyrinthreflexe auf die Augen, welche von der Lage des Kopfes im Raume abhängen, ausschliesslich vom cerebrospinalen Nervensystem abhängen, dass aber andere tonische Zustände der Augenmuskulatur die sympathische Komponente enthalten könnten.

Wegen des eben genannten Gesichtspunktes habe ich eine weitere Reihe von Untersuchungen angestellt: dieselben betrafen den Nachdrehnystagmus welcher eintritt, wenn man Kaninchen auf einer Scheibe rotiert. Zuerst habe ich die normalen Tiere untersucht, sodann die gleichen Tiere nach einseitiger Exstirpation des Sympathicus.

Zur Untersuchung des Nachdrehnystagmus wurden die Tiere auf das Kaninchenbrett festgebunden und auf eine grosse hölzerne Scheibe mit dem Kopf nach der Peripherie zu gebracht. Diese Scheibe habe ich mit der Hand gedreht, und zwar mit einer Geschwindigkeit, dass in 25" 10 Drehungen stattfanden. Im Anfang der Versuche ist die Zahl der Nystagmusbewegungen nach Aufhōren der Drehung eine viel grōssere als wenn man tagelang tăglich 2 mal die Tiere rotiert hat. Es stellt sich nach einigen Tagen eine konstante Zahl der Nystagmusbewegungen ein und erst wenn diese erreicht ist, darf man mit den eigentlichen Versuchen beginnen. Auch in dieser Versuchsreihe habe ich 6 Tiere gebraucht.

Die Ergebnisse dieser Untersuchungen habe ich in Tabelle $\mathrm{E}$ niedergelegt. Sie enthalten die Uebersicht über die Zahlenwerte des Nachdrehnystagmus auf der normalen und auf der sympathicuslosen Seite. Auch diese Reihe hat ein vollständig eindeutiges Resultat. Irgend ein Unterschied zwischen der normalen Seite und der Seite ohne Sympathicus lässt sich nicht konstatieren, und es gelten daher die gleichen Erwägungen, welche ich am Schlusse der vorigen Versuchsreihe angestellt habe. Die tonischen Augenmuskelreflexe wie sie im Nystagmus zur Anschaung gelangen, scheinen keine Komponente $z \mathrm{u}$ enthalten, welehe von sympathischer Innervation herrühren. 
Tabelle E. Untersuchung über den Nachdrehnystagmus bei normalen und sympathicuslosen Kaninchen.

Prüfungsmethode: In 25 Sekunden 10 mal Drehung nach links und rechts.

Jeden Tag 1 oder 2 mal (Vormittag oder Nachmittag) geprüft.

\begin{tabular}{|c|c|c|c|c|c|c|c|}
\hline \multicolumn{8}{|c|}{ 1. Tier. } \\
\hline \multicolumn{4}{|c|}{ Normal } & \multicolumn{4}{|c|}{ Links sympathicuslos. } \\
\hline \multicolumn{2}{|c|}{ Drehung nach 1.} & \multicolumn{2}{|c|}{ Drehung nach r. } & \multicolumn{2}{|c|}{ Drehung nach 1 . } & \multicolumn{2}{|c|}{ Drehung nach r. } \\
\hline $\begin{array}{l}\text { Zahl d. } \\
\text { Nystag. }\end{array}$ & $\begin{array}{l}\text { Dauer d. } \\
\text { Nystag. }\end{array}$ & $\begin{array}{l}\text { Zabl d. } \\
\text { Nystag. }\end{array}$ & $\begin{array}{l}\text { Dauer d. } \\
\text { Nystag. }\end{array}$ & $\begin{array}{l}\text { Zahl d. } \\
\text { Nystag. }\end{array}$ & $\begin{array}{l}\text { Dahl d. } \\
\text { Nystag. }\end{array}$ & $\begin{array}{l}\text { Zabl d. } \\
\text { Nystag. }\end{array}$ & $\begin{array}{l}\text { Dauer d. } \\
\text { Nystag. }\end{array}$ \\
\hline 7 & $6^{\prime \prime}$ & 13 & $7 " \prime$ & 5 & $6 "$ & 6 & $6^{\prime \prime}$ \\
\hline 6 & 6 & 22 & 9 & 4 & 6 & 5 & 5 \\
\hline 6 & 6 & 9 & 6 & 4 & 5 & 7 & 5 \\
\hline 5 & 6 & 8 & 5 & 6 & 6 & 7 & 6 \\
\hline 7 & 7 & 9 & 6 & 4 & 6 & 8 & 6 \\
\hline 10 & 6 & 14 & 7 & 5 & 5 & 6 & 5 \\
\hline 8 & 6 & 13 & 6 & 4 & 5 & 6 & 6 \\
\hline 6 & 5 & 11 & 6 & 5 & 6 & 5 & 5 \\
\hline 5 & 5 & 8 & 5 & 5 & 6 & 6 & 6 \\
\hline 5 & 5 & 6 & 5.5 & 7 & 5 & 7 & 6 \\
\hline
\end{tabular}

2. Tier.

\begin{tabular}{r|l|r|r||r|r|r|r}
\hline 8 & $6 " \prime$ & 7 & $7 \prime \prime$ & 12 & $7 \prime$ & 8 & 5 \\
8 & 6 & 7 & 8 & 10 & 6 & 8 & 5 \\
8 & 6 & 10 & 9 & 8 & 6 & 8 & 5 \\
15 & 6 & 12 & 6 & 10 & 6 & 7 & 5 \\
13 & 7 & 9 & 6 & 9 & 6 & 7 & 6 \\
8 & 6 & 9 & 6 & 8 & 6 & 8 & 5 \\
10 & 6 & 10 & 5 & 7 & 5 & 6 & 5 \\
10 & 5 & 8 & 5 & 10 & 6 & 9 & 5 \\
10 & 5 & 8 & 5 & 6 & 5 & 6 & 5 \\
& & & & 5 & 5 & 6 & 5 \\
\hline
\end{tabular}

3. Tier.

\begin{tabular}{l|l|r|r||r|r|r|l}
\hline 34 & $14 \prime \prime$ & 10 & $12 \prime \prime$ & 12 & $10^{\prime \prime}$ & 13 & $7 \prime \prime$ \\
35 & 16 & 16 & 10 & 10 & 8 & 14 & 9 \\
18 & 14 & 14 & 10 & 16 & 11 & 11 & 8 \\
10 & 12 & 8 & 12 & 13 & 8 & 10 & 8 \\
17 & 12 & 9 & 10 & 10 & 9 & 9 & 9 \\
11 & 10 & 7 & 8 & 8 & 8 & 12 & 9 \\
11 & 10 & 10 & 7 & & & & \\
15 & 12 & 11 & 10 & & & &
\end{tabular}

Bei dieser Prüfung nimmt die Dauer und die Zahl des Nystagmus allmăhlich ab, und nach 7-8 maliger Untersuchung werden sie konstant. Hierauf wird die Exstirpation des Sympathicus vorgenommen. Nystagmus tritt auf beiden Seitẹn gleichzeitig und nach der gleichen Richtung ein. 
3. Tier.

\begin{tabular}{|c|c|c|c|c|c|c|c|}
\hline \multicolumn{4}{|c|}{ Normal } & \multicolumn{4}{|c|}{ Normal } \\
\hline \multicolumn{2}{|c|}{ Drehung nach 1.} & \multicolumn{2}{|c|}{ Drehung nach r. } & \multicolumn{2}{|c|}{ Drehung nach 1 . } & \multicolumn{2}{|c|}{ Drehung nach r. } \\
\hline Zahl & Dauer & Zahl & Dauer & Zahl & Dauer & Zahl & Dauter \\
\hline 11 & $11^{\prime \prime}$ & 12 & $9 \prime \prime$ & 21 & 16 & 11 & $10^{\prime \prime}$ \\
\hline 13 & 13 & 9 & 11 & 21 & 15 & 9 & 10 \\
\hline 10 & 10 & 9 & 9 & 17 & 12 & 8 & 8 \\
\hline
\end{tabular}

4. Tier.

\begin{tabular}{|c|c|c|c|c|c|c|c|}
\hline \multicolumn{4}{|c|}{ Normal } & \multicolumn{4}{|c|}{ Liniks sympathicuslos. } \\
\hline 38 & $14 \prime \prime$ & 31 & $11 " \prime$ & 1 & $2^{\prime \prime}$ & 0 & 0 \\
\hline 34 & 13 & 31 & 12 & 0 & 0 & 0 & 0 \\
\hline 26 & 12 & 18 & 10 & 0 & 0 & 0 & 0 \\
\hline 17 & 9 & 15 & 9 & 1 & 2 & 0 & 0 \\
\hline 17 & 10 & 12 & 8 & 1 & 2 & 0 & 0 \\
\hline 16 & 11 & 14 & 8 & 0 & 0 & 0 & 0 \\
\hline 18 & 11 & 15 & 8 & 0 & 0 & 0 & 0 \\
\hline 9 & 7 & 12 & 8 & & & & \\
\hline 10 & 6 & 15 & 8 & & & & \\
\hline 8 & 7 & 13 & 7 & & & & \\
\hline 2 & 4 & 3 & 4 & & & & \\
\hline 2 & 4 & 2 & 4 & & & & \\
\hline 0 & 0 & 3 & 3 & & & & \\
\hline 1 & 2 & 0 & 0 & & & & \\
\hline 0 & 0 & 0 & 0 & & & & \\
\hline
\end{tabular}

5. Tier.

\begin{tabular}{r|r|r|r||r|r|r|r}
\hline 16 & $9 \prime \prime$ & 36 & $13 \prime \prime$ & 9 & $7 \prime \prime$ & 10 & $7 \prime \prime$ \\
20 & 10 & 34 & 12 & 10 & 7 & 9 & 7 \\
29 & 13 & 33 & 13 & 9 & 7 & 9 & 6 \\
19 & 11 & 25 & 12 & 8 & 7 & 9 & 6 \\
24 & 10 & 19 & 8 & 10 & 6 & 11 & 7 \\
13 & 9 & 19 & 9 & 10 & 7 & 12 & 8 \\
21 & 10 & 17 & 9 & 13 & 7 & 6 & 5 \\
17 & 8 & 12 & 6 & 10 & 6 & 10 & 5 \\
13 & 8 & 13 & 7 & 12 & 7 & 11 & 6 \\
12 & 7 & 13 & 8 & 11 & 7 & 7 & 6 \\
10 & 6 & 10 & 6 & 10 & 7 & 8 & 6 \\
10 & 7 & 10 & 6 & 11 & 7 & 9 & 6 \\
10 & 7 & 9 & 6 & & & & \\
10 & 7 & 10 & 7 & & & & \\
11 & 7 & 11 & 6 & & & & \\
11 & 7 & 12 & 6 & & & & \\
\hline
\end{tabular}




\section{Tier.}

\begin{tabular}{|c|c|c|c|c|c|c|c|}
\hline \multicolumn{4}{|c|}{ Normal } & \multicolumn{4}{|c|}{ Symyathicuslos. } \\
\hline \multicolumn{2}{|c|}{ Drehung nach 1.} & \multicolumn{2}{|c|}{ Drehung nach r. } & \multicolumn{2}{|c|}{ Drehung nach $\mathrm{I}$. } & \multicolumn{2}{|c|}{ Drehung nach $r$. } \\
\hline Zahl & Dauer & Zahl & Dauer & Zahl & Dauer & Zahl & Dauer \\
\hline 31 & $15^{\prime \prime}$ & 35 & $13^{\prime \prime}$ & 9 & $6 "$ & 7 & 6 \\
\hline 54 & 17 & 30 & 11 & 7 & 6 & 6 & 6 \\
\hline 41 & 15 & 31 & 11 & 8 & 6 & 8 & 6 \\
\hline 20 & 12 & 30 & 9 & 8 & 6 & 7 & 6 \\
\hline 26 & 12 & 34 & 9 & 8 & 6 & 7 & 6 \\
\hline 27 & 12 & 27 & 8 & 9 & 6 & 7 & 6 \\
\hline 21 & 10 & 11 & 8 & 8 & 6 & 8 & 6 \\
\hline 16 & 9 & 12 & 7 & 8 & 6 & 7 & 6 \\
\hline 18 & 9 & 14 & 8 & 9 & 6 & 7 & 6 \\
\hline 16 & 11 & 11 & 6 & & & & \\
\hline 14 & 8 & 11 & 7 & & & & \\
\hline 11 & 7 & 11 & 7 & & & & \\
\hline 10 & 7 & 9 & 6 & & & & \\
\hline 10 & 6 & 8 & 5 & & & & \\
\hline 9 & 5 & 10 & 6 & & & & \\
\hline 8 & 5 & 10 & 6 & & & & \\
\hline 9 & 6 & 7 & 6 & & & & \\
\hline 9 & 6 & 8 & 6 & & & & \\
\hline 8 & 6 & 11 & 6 & & & & \\
\hline g & 6 & 8 & 6 & & & & \\
\hline 8 & 6 & 11 & 6 & & & & \\
\hline 8 & 6 & & & & & & \\
\hline
\end{tabular}

(Schluss folgt.) 